\title{
Alterations in upper airway cross-sectional area in response to lower body positive pressure in healthy subjects
}

\author{
Satomi Shiota, Clodagh M Ryan, Kuo-Liang Chiu, Pimon Ruttanaumpawan, James Haight, Michael \\ Arzt, John S Floras, Christopher Chan, T Douglas Bradley
}

See end of article for authors' affiliations

Correspondence to: Dr T Douglas Bradley, Toronto General Hospital/ University Health Network 9N-943, Toronto, Ontario, M5G 2C4, Canada; douglas.bradley@utoronto. $\mathrm{ca}$

Received 30 August 2006 Accepted 1 April 2007 Published Online First 18 April 2007

\begin{abstract}
Background: Fluid accumulation in the neck during recumbency might narrow the upper airway (UA) and thereby contribute to its collapse in patients with obstructive sleep apnoea (OSA). It is hypothesised that acute fluid shifts from the legs to the upper body in healthy subjects would increase neck circumference and reduce the cross-sectional area of the UA (UA-XSA).

Methods: In 27 healthy non-obese subjects of mean (SE) age 39 (3) years and body mass index 23.2 $(0.6) \mathrm{kg} / \mathrm{m}^{2}$ studied while supine, leg fluid volume was measured using bioelectrical impedance, neck circumference using a mercury strain gauge and mean UA-XSA between the velum and the glottis using acoustic pharyngometry at end expiration. Measurements were made at baseline affer which subjects were randomly assigned to a $5 \mathrm{~min}$ time control period or to a $5 \mathrm{~min}$ application of lower body positive pressure (LBPP) at $40 \mathrm{~mm} \mathrm{Hg}$ by anti-shock trousers, separated by a $15 \mathrm{~min}$ washout period. Subjects then crossed over to the opposite arm of the study.

Results: Compared with control, application of LBPP significantly reduced leg fluid volume $(p<0.001)$ and increased neck circumference $(\mathrm{p}<0.001)$, both at $1 \mathrm{~min}$ and $5 \mathrm{~min}$, and reduced UA-XSA after both $1 \mathrm{~min}$ $\left(-0.15 \mathrm{~cm}^{2} ; 95 \% \mathrm{Cl}-0.23\right.$ to $\left.-0.09, \mathrm{p}<0.001\right)$ and $5 \min \left(-0.20 \mathrm{~cm}^{2} ; 95 \% \mathrm{Cl}-0.33\right.$ to -0.09 , $\mathrm{p}<0.001$ ).

Conclusion: In healthy subjects, displacement of fluid from the legs by LBPP causes distension of the neck and narrowing of the UA lumen. Fluid displacement from the lower to the upper body while recumbent may contribute to pharyngeal narrowing and obstruction to airflow in patients with OSA. This may have particular pathological significance in oedematous states such as heart and renal failure.
\end{abstract}

body $^{9}$ and increases central venous pressure, ${ }^{10}$ but without increasing left ventricular filling pressure. ${ }^{11}$

In a recent study we applied LBPP to healthy euvolaemic subjects to mimic the effects of fluid redistribution from the lower to the upper body upon assuming the recumbent position in patients with fluid overload. We showed in 11 healthy subjects that LBPP caused significant increases in neck circumference and pharyngeal resistance to airflow measured by pressure transducers in the hypopharynx and nasopharynx. ${ }^{12}$ However, UA calibre was not measured during those experiments because it could not be assessed concurrently with UA resistance. To test the hypothesis that the increase in resistance we observed was related to narrowing of the UA, we have undertaken a separate study to examine the effects of fluid redistribution from the legs to the neck by LBPP on UA-XSA measured by acoustic pharyngometry. Of the 27 subjects taking part in the present experiments, only 5 took part in our previous study $^{12}$ so the overlap of subjects was minimal. study has examined the effect of shifting fluid from the lower extremities to the upper body on UA size. In that study, Shepard and colleagues ${ }^{8}$ tested the effects of shifting fluid out of the lower body by raising the legs, and of shifting fluid into the legs by applying tourniquets around the thighs in an attempt to displace fluid into and out of the neck, respectively, in patients with OSA. Using CT scanning, they observed a tendency for the UA cross-sectional area (UA-XSA) to decrease and to increase in response to leg raising and tourniquet application, respectively. However, these changes in UA-XSA were not significant, perhaps because these interventions did not cause sufficient fluid displacement to alter UA size. A more effective means of displacing fluid from the lower to the upper body may be application of lower body positive pressure (LBPP) via anti-shock trousers. LBPP displaces fluid into the upper

\section{METHODS}

Subjects

Eligible subjects were healthy men and women, 18 years of age or older, with no history of smoking, cardiovascular, respiratory or neurological disease. Exclusion criteria were obesity (body mass index $(\mathrm{BMI})>30 \mathrm{~kg} / \mathrm{m}^{2}$ ), a history of habitual snoring or daytime sleepiness, taking prescribed medications and pregnancy. The protocol was approved by the local research ethics

Abbreviations: $\mathrm{BMI}$, body mass index; $\mathrm{EELV}$, end expiratory lung volume; LBPP, lower body positive pressure; OSA, obstructive sleep apnoea; UA, upper airway; UA-XSA, upper airway cross-sectional area 
board and subjects gave their written informed consent prior to participating.

\section{Lower body positive pressure (LBPP)}

LBPP was applied using medical anti-shock trousers (MAST IIIAT, David Clark, Worcester, Massachusetts, USA) that were fitted around the subjects' legs from the ankles to the hips in the deflated state. During experiments only the leg bladders were inflated to $40 \mathrm{~mm} \mathrm{Hg}$.

\section{Leg fluid volume}

Leg fluid volume was measured using a bioelectrical impedance spectrum analyser (Xitron Hydra ECF/ICF, Model 4200; Xitron Technologies Inc, San Diego, California, USA). ${ }^{13}$ This is a well validated technique that measures electrical impedance between two pairs of electrodes placed on the body. Changes in impedance are proportional to changes in fluid volume. ${ }^{14} 15$ Electrodes were placed on the ankle and upper thigh to measure fluid volume in one lower limb.

\section{Neck circumference}

Percentage changes in neck circumference during experiments were monitored continuously by a mercury-in-silastic strain gauge (D E Hokanson Inc, Bellevue, Washington, USA) whose resistance to electrical flow is altered in proportion to the change in its length. ${ }^{16}$ The gauge was placed above the cricothyroid cartilage. This device is ordinarily used to measure changes in cross-sectional area of limbs for determination of blood flow and is very sensitive, accurate and reproducible, as we have previously shown. ${ }^{17}$

\section{Upper airway cross-sectional area (UA-XSA)}

Acoustic pharyngometry (Eccovision; Hood Laboratories; Pembroke, Massachusetts, USA) was used to determine UAXSA. ${ }^{18-20}$ With subjects supine and their heads in the neutral position, the device was positioned in the mouth using a mouthpiece designed to secure the tongue in place. The pulse emitter produced five pulses per second. Two microphones detected the amplitude and temporal changes of the reflected pulse. Data were collected and analysed by a single investigator. During the measurements the subject's head position was fixed by resting the head in form-shaping sand bags. UA-XSA area was determined at end expiration as the mean area between the nasal and oropharyngeal junction (velum) and the glottis as previously described..$^{21}$ The mean of four consecutive measurements was used. Acoustic pharyngometry has been validated against CT scanning ${ }^{22}$ and MRI $^{23}$ for assessment of the UA-XSA in non-snorers and in snorers with and without OSA in both the supine and upright positions. ${ }^{21}{ }^{24-26}$

\section{End expiratory lung volume (EELV) and blood pressure}

In six subjects the change in end expiratory lung volume (EELV) was monitored by a respiratory inductance plethysmograph (Respitrace; Ambulatory Monitoring Inc, Ardsley, New York, USA) in the DC coupled mode calibrated against a spirometer. Systolic and diastolic blood pressures were measured using an automated sphygmomanometer (Dinamap 1846SX NIBP, Critikon, Tampa, Florida, USA) on the upper arm. Heart rate was measured during blood pressure measurements.

\section{Protocol}

Following a 5 min stabilisation period, baseline measurements of all variables were made. Subjects were then randomised to a 5 min LBPP exposure or a 5 min control period, at the end of which baseline measurements were repeated. This was followed by a 15 min washout period during which subjects were seated upright. They then returned to the supine position and underwent a second $5 \mathrm{~min}$ baseline period after which they crossed over to the other arm of the study for $5 \mathrm{~min}$. Measurements of all variables were made at the end of each baseline period and after 1 and 5 min during each of the LBPP and control periods. Reproducibility of UA-XSA measurements was determined from five separate measurements in each subject during baseline before the control period, 1 and $5 \mathrm{~min}$ during the control period, and at 1 and 5 min after the control period.

\section{Data analysis}

All variables were analysed at the ends of the baseline period and after 1 and 5 min of the LBPP and control periods. Data from five consecutive breaths at the end of each of these periods were analysed and averaged to provide changes in neck circumference and in EELV from baseline. Two-way repeated measures analysis of variance (ANOVA; Sigma Stat 2.0, SPSS Inc, Chicago, Illinois, USA) was used to compare values obtained during the baseline, control and LBPP periods, followed by a post hoc Tukey test as appropriate. For helium dilution lung volumes, paired $t$ tests were used to compare values during the control and LBPP periods. All data were normally distributed and are presented as mean (SE) values unless stated otherwise. A two-sided $p$ value of $<0.05$ was considered significant.

\section{RESULTS}

\section{Characteristics of subjects}

The demographic data of the 27 study subjects ( 16 men) are summarised in table 1 . They were generally young non-obese adults.

\section{Leg fluid volume and neck circumference}

Table 2 shows that the leg fluid volume did not change significantly after 1 and $5 \mathrm{~min}$ during the control period. In contrast, after 1 and 5 min of LBPP there was a sustained decrease in leg fluid volume compared with baseline that was significantly greater than during 1 and $5 \mathrm{~min}$ of the control period (difference from control -0.161 (95\% confidence interval $(\mathrm{CI})-0.19$ to -0.12$)$ and $-0.14 \mathrm{l}$ (95\% CI -0.18 to -0.09 ), respectively). Neck circumference did not change significantly from baseline during the control period at either 1 or 5 min. In contrast, after 1 and 5 min of LBPP there was a sustained increase in neck circumference compared with baseline that was significantly greater than at 1 and 5 min of the control period (difference from control $0.34 \%$ (95\% CI $0.23 \%$ to $0.45 \%)$ and $0.28 \%(95 \%$ CI $0.12 \%$ to $0.43 \%)$, respectively).

\section{Upper airway cross-sectional area}

Figure 1 shows tracings of UA-XSA as a function of distance from the mouth opening at the end of the baseline period and after 1 and 5 min of LBPP from a representative subject. It shows that LBPP reduced UA-XSA at both $\mathrm{l}$ and $5 \mathrm{~min}$. Measurements of UA-XSA performed on five separate occasions

\begin{tabular}{|ll|}
\hline Table 1 & Characteristics of study subjects \\
\hline Number (M/F) & $27(16 / 11)$ \\
Age (years) & $39(3)$ \\
Height $(\mathrm{cm})$ & $167.5(1.8)$ \\
Weight $(\mathrm{kg})$ & $65.5(2.4)$ \\
BMl $\left(\mathrm{kg} / \mathrm{m}^{2}\right)$ & $23.2(0.6)$ \\
Neck circumference $(\mathrm{cm})$ & $36.5(0.7)$ \\
\hline Data are mean (SE). & \\
BMl, body mass index. & \\
\hline
\end{tabular}


Table 2 Influence of lower body positive pressure (LBPP) on physiological variables

\begin{tabular}{|c|c|c|c|c|}
\hline & Baseline & $1 \mathrm{~min}$ & $5 \mathrm{~min}$ & $\begin{array}{l}\text { p Value for time } \\
\times \text { treatment } \\
\text { interaction }\end{array}$ \\
\hline \multicolumn{5}{|c|}{ Leg fluid volume (I) } \\
\hline Control & $4.67(0.18)$ & $4.67(0.18)$ & $4.66(0.18)$ & \\
\hline LBPP & $4.57(0.19)^{*}$ & $4.41(0.19)^{*} \dagger$ & $4.43(0.19)^{*} \dagger$ & $<0.001$ \\
\hline \multicolumn{5}{|c|}{$\begin{array}{l}\text { Change in neck circumference } \\
\text { (\%) }\end{array}$} \\
\hline Control & - & $-0.01(0.02)$ & $0.00(0.05)$ & \\
\hline LBPP & - & $0.33(0.05)^{\star} \dagger$ & $0.28(0.06)^{*} \dagger$ & $<0.001$ \\
\hline \multicolumn{5}{|c|}{ Change in EELV (l)‡ } \\
\hline Control & - & $-0.024(0.024)$ & $0.107(0.044)$ & \\
\hline LBPP & - & $0.112(0.049)$ & $0.198(0.106)$ & 0.22 \\
\hline \multicolumn{5}{|c|}{$\mathrm{SBP}(\mathrm{mm} \mathrm{Hg}) \ddagger$} \\
\hline Control & $119(10)$ & $120(9)$ & $115(8)$ & \\
\hline LBPP & $122(10)$ & $124(9)$ & $121(9)$ & 0.61 \\
\hline \multicolumn{5}{|c|}{$\mathrm{DBP}(\mathrm{mm} \mathrm{Hg}) \ddagger$} \\
\hline Control & 71 (3) & $70(4)$ & $71(4)$ & \\
\hline LBPP & 74 (4) & $74(4)$ & 75 (4) & 0.62 \\
\hline \multicolumn{5}{|c|}{ Heart rate $(b p m) \ddagger$} \\
\hline Control & $68(6)$ & $67(6)$ & $66(6)$ & \\
\hline LBPP & $68(6)$ & $68(6)$ & $66(6)$ & 0.29 \\
\hline
\end{tabular}

EELV, end expiratory lung volume; SBP, systolic blood pressure; DBP, diastolic blood pressure.

Data are mean (SE).

${ }^{*} p<0.001$ vs control by Tukey test.

$\mathrm{tp}<0.001$ vs baseline by Tukey test.

$\ddagger \mathrm{n}=6$.

under control conditions in each of the 27 subjects were highly reproducible with a mean coefficient of variation of 3.74 $(0.78) \%$.

Figure 2 shows grouped data for all 27 subjects. It illustrates that UA-XSA did not change significantly during the control period at either 1 or $5 \mathrm{~min}$ (from $2.66(0.13)$ to $2.62(0.13) \mathrm{cm}^{2}$, $\mathrm{p}=0.440$; and to $\left.2.60(0.12) \mathrm{cm}^{2}, \mathrm{p}=0.146\right)$. In contrast, after 1 and $5 \mathrm{~min}$ of LBPP there was a sustained reduction in UAXSA (from $2.68(0.13)$ to $2.47(0.12) \mathrm{cm}^{2}, \mathrm{p}<0.001$; and to 2.40 $\left.(0.12) \mathrm{cm}^{2}, \mathrm{p}<0.001\right)$ that was significantly greater than during $\mathrm{l}$ and $5 \mathrm{~min}$ of the control period (difference from control $-0.15 \mathrm{~cm}^{2}(95 \% \mathrm{CI}-0.23$ to -0.09$)$ and $-0.20 \mathrm{~cm}^{2}(95 \%$ CI -0.33 to -0.09 ), respectively). There was also a significant inverse correlation between the magnitude of change in UA-XSA

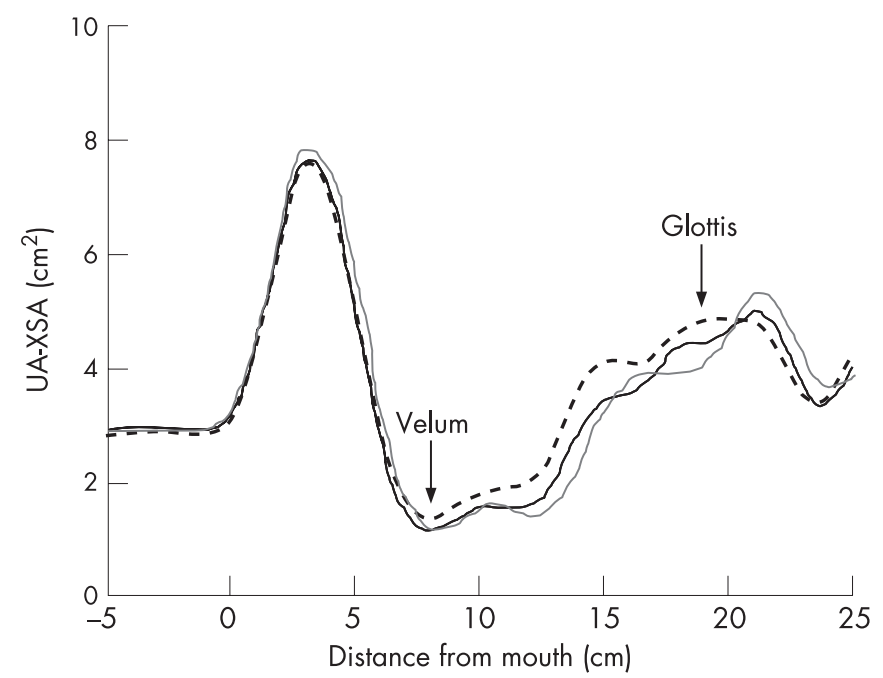

Figure 1 Tracing of upper airway cross-sectional area (UA-XSA) as a function of distance from the mouth in a representative subject. UA-XSA is the mean area between the velum and glottis as indicated by the arrows. Note that, compared with baseline (dashed line, $2.83 \mathrm{~cm}^{2}$ ), lower body positive pressure application caused a reduction in UA-XSA after $1 \mathrm{~min}$ (solid line, $2.44 \mathrm{~cm}^{2}$ ) and $5 \mathrm{~min}$ (grey line, $2.37 \mathrm{~cm}^{2}$ ). and neck circumference at $1 \mathrm{~min}(\mathrm{r}=-0.53, \mathrm{p}=0.01)$ but there was no significant correlation between the change in UA-XSA and neck circumference after $5 \min (r=-0.15, \mathrm{p}=0.50)$.

\section{EELV, blood pressure and heart rate}

Table 2 shows that EELV, systolic and diastolic blood pressures and heart rate did not change significantly from baseline during either the control or LBPP periods. There were no side effects during the application of LBPP.

\section{DISCUSSION}

In this study of healthy non-obese subjects, we have made several interesting and novel observations. First we have shown that application of $40 \mathrm{~mm} \mathrm{Hg}$ of LBPP, which displaced approximately $320 \mathrm{ml}$ from both legs (assuming the fluid shift was twice that of one leg), increased the circumference of the neck. This indicated that a portion of this fluid was shifted into the nuchal structures. Second, this distension of the neck was accompanied by a significant $9 \%$ reduction in UA-XSA. Because LBPP caused no significant effect on EELV, blood pressure or heart rate, the most likely explanation for this decrease in UAXSA was fluid accumulation in the nuchal structures, including the great veins and/or soft tissues. These observations show that the LBPP induced increase in UA resistance that we recently described ${ }^{12}$ was related to constriction of the pharynx.

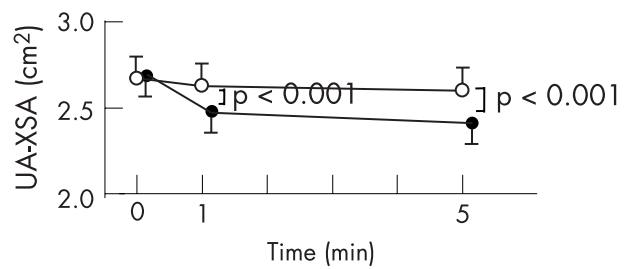

Figure 2 Grouped data showing changes in upper airway cross-sectional area (UA-XSA) in response to lower body positive pressure (LBPP); $p<0.001$ for time $\times$ treatment interaction (two-way repeated measures ANOVA). Compared with the control period, UA-XSA decreased significantly at both 1 and 5 min after applying LBPP. $p$ Values shown in plots are adjusted for multiple comparisons by Tukey test. 
Taken together, these findings may have implications for the pathogenesis of UA obstruction in patients with OSA, particularly in those with coexisting oedematous states. The very low coefficient of variation under control conditions in our 27 subjects also provides evidence of the high reproducibility of UA-XSA measurements using acoustic pharyngometry.

We limited our application of LBPP to $5 \mathrm{~min}$ because we found empirically during pilot studies that 5 min was a time beyond which some subjects began to feel uncomfortable from leg compression while maintaining constant body and head position during measurement of UA-XSA by acoustic pharyngometry. We did not assess the duration of the effect of LBPP on UA-XSA following deflation of the trousers. However, because there was a 15 min washout period between conditions, and because there were no differences in baseline values for the control and LBPP conditions for any variable except for leg fluid volume, we can assume that any potential carryover effect lasted less than $15 \mathrm{~min}$. With respect to leg fluid volume, it was significantly less at baseline during LBPP than at baseline during control. However, since leg fluid volume fell after 1 and 5 min of LBPP, we cannot attribute the higher baseline fluid volume during control to a carryover effect of LBPP.

Although subjects and researchers were not blinded to the control and LBPP conditions, measurements of leg fluid volume, neck circumference and UA-XSA are performed automatically and are therefore not subject to observer interpretation.

The only other study to examine the effect of fluid shift from the legs on UA-XSA, apart from our present and previous ones, ${ }^{12}$ was performed by Shepard and colleagues. ${ }^{8}$ They studied 10 patients with OSA and shifted fluid out of the legs by raising them $33^{\circ}$ from the horizontal, and shifted fluid into the legs by applying venous occlusion tourniquets to the upper thighs for 10 min each. UA-XSA was assessed at baseline and at the end of these two 10 min interventions by CT scanning. However, they did not measure either the amount of fluid displaced out of or into the legs in response to these interventions, nor did they assess whether any fluid was displaced into or out of the neck. They observed a tendency for UA-XSA to decrease in response to leg raising and to increase in response to tourniquet application, but neither of these changes was significant. The reason why they did not find a significant reduction in UA-XSA in response to leg raising may be that the amount of fluid displaced was not sufficient to alter fluid volume in the nuchal structure or to cause neck distension or UA narrowing. For example, using radionuclide scintigraphy, it has been reported that leg raising reduces leg blood volume by $30-35 \%$ or approximately $150 \mathrm{ml}$ or less for both legs. ${ }^{27}{ }^{28}$ This is less than half the volume we displaced using LBPP of $40 \mathrm{~mm} \mathrm{Hg}$. The mean $320 \mathrm{ml}$ displacement of fluid from the legs by LBPP in our study is similar to the $240-360 \mathrm{ml}$ that is displaced from the legs of healthy subjects when moving from standing to the recumbent position, and so is liable to be physiologically and clinically relevant. ${ }^{29}$ It has also been shown in healthy subjects that LBPP displaces fluid into the upper body and increases central venous pressure. ${ }^{10}$ It is therefore possible that fluid influx into the jugular veins might displace the lateral pharyngeal walls medially and contribute to UA narrowing and obstruction to airflow. ${ }^{30}$

Several observations suggest that fluid accumulation in the neck and peripharyngeal soft tissues might have a role in narrowing the UA and increasing its resistance to airflow. For instance, systemic vasodilation in cats by sodium nitroprusside reduces pharyngeal volume and increases UA closing pressure whereas systemic vasoconstriction does the opposite. ${ }^{29}$ In humans, topical application of the vasoconstrictor phenylephrine reduced UA resistance. ${ }^{31}{ }^{32}$ In patients with OSA, surgical specimens from uvulopalatopharyngoplasty revealed vascular congestion and diffuse interstitial oedema. ${ }^{33}$ In another study involving patients with OSA, peripharyngeal oedema apparent on MRI scanning before treatment decreased following chronic application of continuous positive airway pressure. ${ }^{34}$ Two of the present observations support this concept by demonstrating that (1) fluid displacement from the legs into the neck causes UA narrowing and (2) there is a significant inverse correlation between changes in UA-XSA and in neck circumference after 1 min of LBPP. However, this relationship was no longer significant after 5 min of LBPP, possibly because factors other than distension of the neck veins-such as oedema formation-intervened during this time and contributed to this effect.

Obesity and increased neck circumference are important risk factors for OSA, but these only explain about 33\% of the variance in the apnoea-hypopnoea index. ${ }^{2}$ Additional factors that may contribute to severity of OSA could include alterations in UA sensory, reflex and motor control as well as variations in the bony structures surrounding the UA. ${ }^{35}$ The physiological data presented here suggest that fluid retention in generaland in the nuchal structures in particular-might also predispose to UA obstruction. Redistribution of fluid from the lower extremities to the upper body on assuming the recumbent position could narrow the UA overnight during sleep, especially in subjects with fluid retaining states. This might explain in part why OSA is more common in patients with heart and renal failure than in the general population. ${ }^{46}$ It may also help to explain the recent observation by Tang et $a l^{37}$ that removal of $1.47 \mathrm{l}$ of fluid more during the night by nocturnal than by $24 \mathrm{~h}$ peritoneal dialysis caused a $76 \%$ reduction in the frequency of obstructive apnoeas and hypopnoeas during sleep. These authors speculated that this effect was due to removal of excess fluid accompanied by a reduction in UA oedema, although they did not make any measurements of UA properties. In any case, their data are compatible with ours in suggesting that fluid accumulation in the neck and UA structures can contribute to pharyngeal narrowing and collapse.

Acoustic pharyngometry assesses the lumen size of the UA but does not define the structures surrounding the lumen. In addition, because the sound waves are only transmitted through the mouth down the pharyngeal lumen below the velum, we could not evaluate the cross-sectional area of the retropalatal pharynx. Nevertheless, many studies using acoustic pharyngometry have shown consistently that the mean UAXSA from the velum to the glottis in the awake state is narrower in patients with OSA than in those without OSA, in obese and non-obese patients alike, both while upright and supine. ${ }^{24} 38{ }^{39}$ Furthermore, our recent demonstration that LBPP causes a large $102 \%$ increase in UA resistance in health subjects $^{12}$ indicates that the reduction in UA-XSA we observed is physiologically significant.

UA-XSA is to some extent dependent on lung volume. ${ }^{40}$ However, since we have shown that EELV does not change significantly in response to LBPP (indeed, it tended to increase), the decrease in UA-XSA we observed in response to LBPP cannot be explained by a reduction in lung volume. ${ }^{12}$ These findings are consistent with those of Regnard et al ${ }^{41}$ who also showed that application of $60 \mathrm{~mm} \mathrm{Hg}$ LBPP caused no significant change in functional residual capacity or vital capacity in healthy subjects.

Since our measurements were performed while subjects were awake, the present finding may not be entirely applicable to the sleeping state. However, since the UA narrows at sleep onset owing to withdrawal of neural input to the pharyngeal dilator muscles, fluid displacement into the nuchal structures during sleep should cause an even greater degree of narrowing than 
during wakefulness. Our finding that LBPP causes UA narrowing does not necessarily indicate that fluid shift into the nuchal structures increases the propensity of the UA to collapse. Nevertheless, it does indicate that LBPP can increase the degree of obstruction to airflow in the UA. We did not perform sleep studies to ascertain whether subjects had OSA or not. However, it is likely that none or only a very small minority had OSA since none of our subjects was obese, had a history of habitual snoring or daytime sleepiness and they were young (mean age 39 years). Future experiments will be required to determine whether there are differences in the effects of LBPP on UA-XSA between subjects with and without OSA.

In summary, the present data provide novel evidence in healthy non-obese subjects that fluid displacement from the legs into the neck can constrict the UA lumen. Fluid displacement from the lower to the upper body while recumbent may therefore contribute to pharyngeal narrowing and predispose to its collapse during sleep in patients with OSA. This may be of even greater clinical relevance in oedematous states. Further studies will be required to determine whether fluid shifts into and out of the neck facilitate or alleviate, respectively, UA obstruction during sleep in patients with OSA.

\section{Authors' affiliations}

\section{Satomi Shiota, Clodagh M Ryan, Kuo-Liang Chiu, Pimon}

Ruttanaumpawan, Michael Arzt, T Douglas Bradley, Sleep Research Laboratory of the Toronto Rehabilitation Institute, Toronto, Ontario, Canada

John S Floras, Christopher Chan, Departments of Medicine of the Mount Sinai and Toronto General Hospitals of the University Health Network, University of Toronto, Toronto, Ontario, Canada

James Haight, Division of Otolaryngology of the University of Toronto, Toronto, Ontario, Canada

Supported by grants from the Toronto Rehabilitation Institute and the Canadian Institutes of Health Research (MOP 11607). SS and CMR were supported by research fellowships from Toronto Rehabilitation Institute, KLC by research fellowships from China Medical University Hospital, Taichung, Taiwan, ResMed and Respironics Inc, PR by a research fellowship from Siriraj Hospital, Bangkok, Thailand, MA by research fellowships from the German Research Foundation and Heart and Stroke Foundation of Ontario, and JSF by a Canada Research Chair in Integrative Cardiovascular Biology and a Career Investigator Award from the Heart and Stroke Foundation of Ontario.

Competing interests: None.

\section{REFERENCES}

1 Davies RJ, Stradling JR. The relationship between neck circumference, radiographic pharyngeal anatomy, and the obstructive sleep apnoea syndrome. Eur Respir J 1990;3:509-14.

2 Katz I, Stradling J, Slutsky AS, et al. Do patients with obstructive sleep apnea have thick necks? Am Rev Respir Dis 1990;141:1228-31.

3 Javaheri S, Parker TJ, Liming JD, et al. Sleep apnea in 81 ambulatory male patients with stable heart failure. Types and their prevalences, consequences, and presentations. Circulation 1998;97:2154-9.

4 Kuhlmann U, Becker HF, Birkhahn M, et al. Sleep-apnea in patients with endstage renal disease and objective results. Clin Nephrol 2000;53:460-6.

5 Sin DD, Fitzgerald F, Parker JD, et al. Risk factors for central and obstructive sleep apnea in 450 men and women with congestive heart failure. Am J Respir Crit Care Med 1999;160:1101-6.

6 Wadhwa NK, Mendelson WB. A comparison of sleep-disordered respiration in ESRD patients receiving hemodialysis and peritoneal dialysis. Adv Perit Dial 1992:8:195-8.

7 Young T, Palta M, Dempsey J, et al. The occurrence of sleep-disordered breathing among middle-aged adults. N Engl J Med 1993;328:1230-5.

8 Shepard JW Jr, Pevernagie DA, Stanson AW, et al. Effects of changes in central venous pressure on upper airway size in patients with obstructive sleep apnea. Am J Respir Crit Care Med 1996;153:250-4.

9 Bivins HG, Knopp R, Tiernan C, et al. Blood volume displacement with inflation of antishock trousers. Ann Emerg Med 1982;11:409-12.
10 Shi X, Crandall CG, Raven PB. Hemodynamic responses to graded lower body positive pressure. Am J Physiol 1993;265:H69-73.

11 Bain RJ, Tan LB, Murray RG, et al. Central haemodynamic changes during lower body positive pressure in patients with congestive cardiac failure. Cardiovasc Res 1989;23:833-7.

12 Chiu KL, Ryan CM, Shiota S, et al. Fluid shift by lower body positive pressure increases pharyngeal resistance in healthy subjects. Am J Respir Crit Care Med 2006; 174:1378-83.

13 Kushner RF, de Vries PM, Gudivaka R. Use of bioelectrical impedance analysis measurements in the clinical management of patients undergoing dialysis. Am J Clin Nutr 1996;64(Suppl):503-9S.

14 Patel RV, Matthie JR, Withers PO, et al. Estimation of total body and extracellular water using single- and multiple-frequency bioimpedance. Ann Pharmacother 1994;28:565-9.

15 Van Loan MD WP, Matthie J, Mayclin PL. Use of bioimpedance spectroscopy to determine extracellular fluid, intracellular fluid, total body water, and fat-free mass. Basic Life Sci 1993;60:67-70.

16 Hokanson DE, Sumner DS, Strandness DE Jr. An electrically calibrated plethysmograph for direct measurement of limb blood flow. IEEE Trans Biomed Eng 1975;22:25-9.

17 Floras JS, Hara K. Sympathoneural and haemodynamic characteristics of young subjects with mild essential hypertension. J Hypertens 1993;11:647-55.

18 Jackson AC, Butler JP, Millet EJ, et al. Airway geometry by analysis of acoustic pulse response measurements. J Appl Physiol 1977;43:523-36.

19 Fredberg JJ, Wohl ME, Glass GM, et al. Airway area by acoustic reflections measured at the mouth. J Appl Physiol 1980;48:749-58.

20 Benson E. Eccovision acoustic pharyngometry system: operator manual 1996.

21 Kamal I. Normal standard curve for acoustic pharyngometry. Otolaryngol Head Neck Surg 2001;124:323-30.

22 D'Urzo AD, Rubinstein I, Lawson VG, et al. Comparison of glottic areas measured by acoustic reflections vs. computerized tomography. J Appl Physiol 1988:64:367-70.

23 Marshall I, Maran NJ, Martin S, et al. Acoustic reflectometry for airway measurements in man: implementation and validation. Physiol Meas 1993; 14:157-69.

24 Jung DG, Cho HY, Grunstein RR, et al. Predictive value of Kushida index and acoustic pharyngometry for the evaluation of upper airway in subjects with or without obstructive sleep apnea. J Korean Med Sci 2004;19:662-7.

25 Kamal I. Test-retest validity of acoustic pharyngometry measurements. Otolaryngol Head Neck Surg 2004;130:223-8.

$26 \mathrm{Kamal} \mathrm{I}$. Acoustic pharyngometry patterns of snoring and obstructive sleep apnea patients. Otolaryngol Head Neck Surg 2004;130:58-66.

27 Rutlen DL, Wackers FJ, Zaret BL. Radionuclide assessment of peripheral intravascular capacity: a technique to measure intravascular volume changes in the capacitance circulation in man. Circulation 1981;64:146-52.

28 Gaffney FA, Bastian BC, Thal ER, et al. Passive leg raising does not produce a significant or sustained autotransfusion effect. J Trauma 1982;22:190-3.

29 Waterfield RL. The effect of posture on the volume of the leg. J Physiol $1931 ; 72: 121-31$

30 Rodenstein DO, Dooms G, Thomas Y, et al. Pharyngeal shape and dimensions in healthy subjects, snorers, and patients with obstructive sleep apnoea. Thorax 1990;45:722-7.

31 Wasicko MJ, Hutt DA, Parisi RA, et al. The role of vascular tone in the control of upper airway collapsibility. Am Rev Respir Dis 1990;141:1569-77.

32 Wasicko MJ, Leiter JC, Erlichman JS, et al. Nasal and pharyngeal resistance after topical mucosal vasoconstriction in normal humans. Am Rev Respir Dis $1991 ; 144: 1048-52$.

33 Anastassov GE, Trieger N. Edema in the upper airway in patients with obstructive sleep apnea syndrome. Oral Surg Oral Med Oral Pathol Oral Radiol Endod 1998:86:644-7.

34 Ryan CF, Lowe AA, Li D, et al. Magnetic resonance imaging of the upper airway in obstructive sleep apnea before and after chronic nasal continuous positive airway pressure therapy. Am Rev Respir Dis 1991;144:939-44.

35 Ryan CM, Bradley TD. Pathogenesis of obstructive sleep apnea. J Appl Physiol 2005;99:2440-50.

36 Arzt $M$, Young T, Finn L, et al. Sleepiness and sleep structure in heart failure patients with and without obstructive sleep apnea. Arch Intern Med 2006;166:1716-22.

37 Tang SC, Lam B, Ku PP, et al. Alleviation of sleep apnea in patients with chronic renal failure by nocturnal cycler-assisted peritoneal dialysis compared with conventional continuous ambulatory peritoneal dialysis. J Am Soc Nephrol 2006;17:2607-16.

38 Bradley TD, Brown IG, Grossman RF, et al. Pharyngeal size in snorers, nonsnorers, and patients with obstructive sleep apnea. N Engl J Med 1986;315:1327-31.

39 Rivlin J, Hoffstein V, Kalbfleisch J, et al. Upper airway morphology in patients with idiopathic obstructive sleep apnea. Am Rev Respir Dis 1984; 129:355-60

40 Hoffstein V, Zamel N, Phillipson EA. Lung volume dependence of pharyngeal cross-sectional area in patients with obstructive sleep apnea. Am Rev Respir Dis 1984;130:175-8

41 Regnard J, Baudillard P, Salah B, et al. Inflation of antishock trousers increases bronchial response to methacholine in healthy subjects. J Appl Physiol 1990;68:1528-33. 